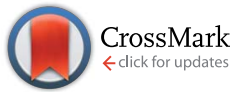

Cite this: Chem. Sci., 2016, 7, 4548

\title{
On the application of the tolerance factor to inorganic and hybrid halide perovskites: a revised system $\uparrow$
}

\begin{abstract}
W. Travis, $\neq^{a}$ E. N. K. Glover, $\star^{a}$ H. Bronstein, ${ }^{a}$ D. O. Scanlon ${ }^{b c}$ and R. G. Palgrave
The tolerance factor is a widely used predictor of perovskite stability. The recent interest in hybrid perovskites for use as solar cell absorbers has lead to application of the tolerance factor to these materials as a way to explain and predict structure. Here we critically assess the suitability of the tolerance factor for halide perovskites. We show that the tolerance factor fails to accurately predict the stability of the 32 known inorganic iodide perovskites, and propose an alternative method. We introduce a revised set of ionic radii for cations that is anion dependent, this revision is necessary due to increased covalency in metal-halide bonds for heavier halides compared with the metal-oxide and fluoride bonds used to calculate Shannon radii. We also employ a 2D structural map to account for the size requirements of the halide anions. Together these measures yield a simple system which may assist in the search for new hybrid and inorganic perovskites.
\end{abstract}

Received 15th December 2015 Accepted 1st April 2016

DOI: $10.1039 / \mathrm{c} 5 \mathrm{sc} 04845 \mathrm{a}$

www.rsc.org/chemicalscience

The perovskite structure can be adopted by compounds of

\section{Introduction}

Predicting the most stable structure for a given chemical composition is an ongoing challenge in chemistry, particularly for solid state non-molecular inorganic compounds. The advent of modern computational methods has significantly advanced our ability to successfully predict the structure of a previously unknown composition. ${ }^{\mathbf{1 , 2}}$ These computational approaches, however, remain time intensive, and are not suitable for all compounds. In contrast, simple geometric approaches to the understanding and prediction of stability in ionic solid state structures have been used for around a century. In such approaches, the constituent ions are assumed to be hard spheres, and, following the methodology of Shannon, ${ }^{3,4}$ their radii can be assumed, with remarkable success, to be constant for a given charge state and coordination number. A simple calculation of ratios of ionic radii can assess whether spheres of a particular size can pack together in a particular structure. The perovskite structure is one of the most widely studied solid state structures, and its understanding has been greatly aided by the use of geometric approaches.

${ }^{a}$ Department of Chemistry, University College London, 20 Gordon Street, London, WC1H 0AJ, UK. E-mail: r.palgrave@ucl.ac.uk

${ }^{b}$ University College London, Kathleen Lonsdale Materials Chemistry, Department of Chemistry, 20 Gordon Street, London, WC1H OAJ, UK

'Diamond Light Source Ltd., Diamond House, Harwell Science and Innovation Campus, Didcot, Oxfordshire, OX11 ODE, UK

$\dagger$ Electronic supplementary information (ESI) available: Lists of metal-anion bond lengths, ABX3 compounds. See DOI: 10.1039/c5sc04845a

$\$$ Authors contributed equally. formula $\mathrm{ABX}_{3}$, where $\mathrm{A}$ and $\mathrm{B}$ are cations and $\mathrm{X}$ is an anion. It is based on a cubic array of corner sharing $\mathrm{BX}_{6}$ octahedra, with the A site cation located within the cuboctahedral cavities. An alternative way to view the structure is of a cubic close packed $\mathrm{AX}_{3}$ array with the $\mathrm{B}$ site cations within the octahedral holes. For the perovskite structure, the most commonly used and most successful geometric ratio is the Goldschmidt tolerance factor, $t$, defined as follows: ${ }^{5}$

$$
t=\frac{r_{\mathrm{A}}+r_{\mathrm{X}}}{\sqrt{2\left(r_{\mathrm{B}}+r_{\mathrm{X}}\right)}}
$$

where $r_{\mathrm{A}}$ and $r_{\mathrm{B}}$ are the ionic radius of the $\mathrm{A}$ and $\mathrm{B}$ site cations respectively, and $r_{\mathrm{X}}$ is the ionic radius of the anion. The tolerance factor assesses whether the A site cation can fit within the cavities in the $\mathrm{BX}_{3}$ framework. A tolerance factor of 1 indicates a perfect fit; in the range $0.8 \leq t \leq 1$ perovskites generally do form, although in the lower part of this range they may be distorted due to tilting of the $\mathrm{BX}_{6}$ octahedra and lowering of the symmetry. If $t>1$, this indicates the A site cation is too large and generally precludes formation of a perovskite, and if $t<0.8$, the A cation is too small, again often leading to alternative structures. The tolerance factor has been very successful in describing and predicting oxide and fluoride perovskite stability, i.e. $\mathrm{ABX}_{3}$ compounds where $\mathrm{X}=\mathrm{O}^{2-}$ or $\mathrm{F}^{-}{ }^{6}$ In these compounds, the high electronegativity of the anions leads to a large degree of ionicity in the bonding, and makes the assumptions of the hard sphere model more valid. In their 2004 review of $\mathrm{ABO}_{3}$ compounds, Li et al. identified $192 \mathrm{ABO}_{3}$ compounds, of which 121 formed perovskites at room temperature and pressure. ${ }^{7}$ Out of 192 compounds, 163 (85\%) were 
categorised correctly as perovskites or non-perovskites using the tolerance factor criterion for perovskite stability $0.8 \leq t \leq 1$. The same authors also found that for $65 \mathrm{ABF}_{3}$ compounds, 62 (95\%) were correctly classified using a criterion for perovskite stability of $t>0.85 .^{8}$

Recently, halide perovskites have attracted very significant attention due to the emergence of hybrid perovskite solar absorbers after an initial report in 2009 by Kojima et al. ${ }^{9}$ Hybrid halide perovskites are compounds of formula $\mathrm{ABX}_{3}$ where the $\mathrm{A}$ site is occupied by a small organic cation, such as methylammonium, $\mathrm{CH}_{3} \mathrm{NH}_{3}{ }^{+}$and $\mathrm{X}$ is a halide anion. These materials are able to absorb light and separate the resulting charge carriers with remarkable efficiency, yet can be produced through simple, bench top chemistry, and are presently the most exciting of the emerging solar cell technologies. ${ }^{\mathbf{1 0 - 1 4}}$ The most efficient hybrid solar cell materials use iodide on the $\mathrm{X}$ site of a perovskite structure, as this leads to a band gap close to the optimum for single junction PV cells. $\mathrm{CH}_{3} \mathrm{NH}_{3} \mathrm{PbI}_{3}$ has a band gap of around $1.5 \mathrm{eV}$ and is the most promising of the hybrid PV solar absorber materials. ${ }^{15}$ Substituting iodide with lighter halides, or adoption of alternative $\mathrm{ABX}_{3}$ structures such as hexagonal perovskite, both lead to a widening of the bandgap and concurrent decrease of the PV efficiency. ${ }^{16}$ Prior to the discovery of hybrid solar cells, hybrid iodide perovskites and indeed purely inorganic iodide perovskites had been studied for many years in relation to several diverse applications. ${ }^{17-24}$

Due to the very rapid and dramatic advance of the hybrid perovskite solar cell field, there is a great motivation to find new hybrid halide perovskite compounds, ${ }^{\mathbf{1 2 , 2 5}}$ and in order to predict compositions that will form stable perovskite structures, the tolerance factor has been widely employed. ${ }^{\mathbf{2 6 - 2 8}}$ However, caution is necessary, as a number of the assumptions underlying any geometric approach to predicting solid state structures must be questioned for the case of the hybrid halide perovskites:

(1) The organic cations are non-spherical, and so an obvious difficulty is encountered in defining the A site ionic radius for use in eqn (1).

(2) Due to the low decomposition temperatures of the organic molecular ions that occupy the A site, hybrid perovskites tend to be produced using low temperature syntheses, meaning that kinetic trapping of less thermodynamically stable structures is possible.

(3) The lower electronegativity of the heavier halides and greater chemical softness, especially of the iodide anion, compared with oxides and fluorides means that the assumption that the ions are unpolarisable hard spheres is less valid.

(4) The tables of cation ionic radii composed by Shannon et al. are taken from oxide and fluoride compounds only, therefore it necessary to question how well they apply to the heavier halides.

The severity of points 3 and 4 is expected to increase moving from chloride to iodide anions. Yet it is the iodides (and to some extent bromides) which are of current technological interest as PV absorber materials, because, as already mentioned, the heavier halides result in compounds with optimal band gaps for solar absorption. Predicting the stability of the heavier hybrid halide perovskites is of most pressing need, and is also expected to be most challenging given the chemical differences between the heavier and lighter halides mentioned in points 3 and 4 above.

For this reason we will first critically examine the applicability of tolerance factor and other geometric criteria to the prediction of hybrid iodide perovskite stability. Cheetham and co-workers have suggested that the range of stability for hybrid iodide perovskites is roughly $0.8 \leq t \leq 1$, i.e. very similar to that found for the oxides and fluorides. ${ }^{26,27}$ This makes intuitive sense as the stability limits are based on geometry rather than any chemical properties, so in principle might be assumed to be universal. We note that whilst points 1 and to an extent 2 (above) apply specifically to hybrid perovskites, points 3 and 4 apply equally to inorganic iodide perovskites too, i.e. compounds in which A is a simple inorganic cation. As a starting point, therefore, we have tested the tolerance factor criterion $0.8 \leq t \leq 1$ against the known inorganic $\mathrm{ABI}_{3}$ compounds. A search of the Inorganic Crystal Structure Database (ICSD), supplemented by a general literature search, revealed 32 crystallographically characterised inorganic $\mathrm{ABI}_{3}$ compounds. Of these, eight formed perovskite structures at room temperature and pressure $\left(\mathrm{RbDyI}_{3}, \mathrm{RbTmI}_{3}, \mathrm{CsCaI}_{3}, \mathrm{RbSnI}_{3}, \mathrm{CsSnI}_{3}, \mathrm{CsDyI}_{3}\right.$, $\left.\mathrm{CsYbI}_{3}, \mathrm{CsPbI}_{3}\right),{ }^{21-23,29-36}$ whilst 24 did not (see ESI Table S3† for a lookup table of $\mathrm{ABI}_{3}$ references sorted by $\mathrm{A}$ and $\mathrm{B}$ cation). Compounds are categorised as a perovskites if the structure is based on a cubic close packed $\mathrm{AX}_{3}$ sublattice, i.e. their $\mathrm{BX}_{3}$ sublattice consists exclusively of corner sharing octahedra connected in three dimensions. The prototypical perovskite structure in the $P m \overline{3} m$ space group, and structures related to this through tilting of the $\mathrm{BX}_{6}$ octahedra or off centring of the $\mathrm{A}$ site cation are therefore included as perovskites. Compounds commonly referred to as hexagonal perovskites, where some proportion of the $\mathrm{AX}_{3}$ sublattice adopts hexagonal close packing, and where some degree of $\mathrm{BX}_{6}$ octahedra edge sharing is present, are listed as non-perovskites for the purposes of this discussion, since these compounds exhibit much larger band gaps than their perovskite counterparts, making them generally unsuitable for PV applications.

We use the Shannon radii as employed by Cheetham et al. and others ${ }^{8,26,27}$ to calculate $t$ for each of the known purely inorganic $\mathrm{ABI}_{3}$ compounds. Fig. 1 shows the distribution of

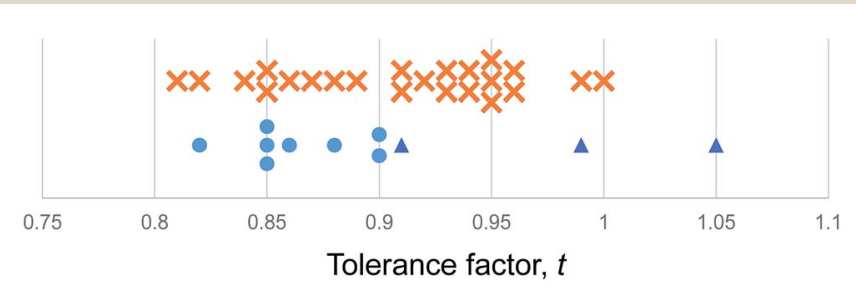

Fig. 1 The tolerance factors of $\mathrm{ABI}_{3}$ compounds. Blue dots represent inorganic $\mathrm{ABI}_{3}$ compounds that form perovskites at room temperature and pressure. Red crosses represent inorganic $\mathrm{ABI}_{3}$ compounds that do not form perovskites. Blue triangles show hybrid $\mathrm{APbl}_{3}$ and $\mathrm{ASnl}_{3}$ compounds that form in the perovskite structure, using the methodology of Cheetham et al. to estimate molecular ion radii. ${ }^{26,27}$ There is no boundary on the tolerance factor scale that separates perovskites from non-perovskites. 
tolerance factors, $t$, calculated for these compounds and expressed to two decimal places. Blue dots represent perovskites and red crosses represent non perovskites. As can be seen, all 32 compounds fall within the range $0.8 \leq t \leq 1$, so we conclude that this criterion is not useful for predicting or explaining structure stability.

Using the tolerance factor alone, the best empirical criterion that can be applied to this set of known inorganic $\mathrm{ABI}_{3}$ compounds is perovskite stability in the range $0.8 \leq t \leq 0.9$. Within this range, all eight of the known perovskite compounds are found. However, nine out of 24 non perovskites are also in this region. Thus using a perovskite stability criterion of $0.8 \leq t$ $\leq 0.9,72 \%$ of inorganic $\mathrm{ABI}_{3}$ compounds are categorised correctly. This figure is lower than can be achieved for the oxides and fluorides, but furthermore this second criterion is less satisfying, as when hybrid compounds are considered, which have large A site cations and therefore large tolerance factors, such a criterion would predict that no hybrid perovskites are stable, which is not the case.

We conclude from the above analysis that having a tolerance factor within a specific range (calculated from traditional Shannon radii) may be a necessary condition for perovskite formation but it is not a sufficient condition, and the traditional approach that works reasonably effectively for fluoride and oxide compounds cannot be used to explain the known structures of the inorganic $\mathrm{ABI}_{3}$ compounds. Given the additional difficulties pertinent to prediction of hybrid structures, over and above those encountered for the purely inorganic iodides, we suggest that there is no reason to expect this approach as it stands would be successful in predicting the stability or otherwise new hybrid perovskite structures.

A question then presents itself: is it possible to use geometric methods to understand and predict halide perovskite stability in general and hybrid iodide perovskite stability in particular? Here we cautiously answer in the affirmative by introducing an adapted approach that takes into account the chemical and physical differences between the heavier halides and the fluoride and oxide anions for which the tolerance factor approach is successful. Using this approach we are able to draw a structure map with simple criteria for halide perovskite stability that gives a success rate over $92 \%$ for the library of inorganic halide perovskites complied by Li et al. ${ }^{8}$ The hybrid perovskites present further challenges but broadly fit within the stability framework of their inorganic counterparts. We believe the concepts we set out below could be extended to other halide structures as well.

\section{Approach}

Structures containing chloride, bromide or iodide anions have several important chemical differences compared with fluorides or oxides for which the concept of Shannon radii and tolerance factors were originally developed. Firstly, the anion is now larger. The iodide anion has a Shannon radius of $2.20 \AA$, compared to $1.28 \AA$ for fluoride and $1.35 \AA$ for oxide. Secondly, the heavier halides are less electronegative: I is 2.66 on the Pauling electronegativity scale compared with $\mathrm{O}$ at 3.16 and $\mathrm{F}$ at 3.98. This means bonds between heavier halides and metals will tend to have greater covalency, which should increase down the halogen group, and the model of hard spheres will be less applicable. We propose two main adaptations that are necessary from the procedure used with oxides and fluorides.

Each of these points will be elaborated on below:

(1) Revised cation radii: for heavier halides, a different set of cation ionic radii must be used for the $\mathrm{p}, \mathrm{d}$ and $\mathrm{f}$ block metals to account for the deviations from Shannon radii.

(2) Additional geometric considerations: a suitable tolerance factor is a necessary but not a sufficient condition for formation of the perovskite structure by $\mathrm{ABX}_{3}$ compounds, where $\mathrm{X}=\mathrm{Cl}$, $\mathrm{Br}$, I. This is due to the larger size of the anion which makes other geometric considerations, especially the ability to octahedrally coordinate a given metal cation, of equal importance to the tolerance factor in determining perovskite stability.

\section{Revised cation radii}

A point not always noted is that the widely used Shannon cation radii are calculated from oxide and fluoride compounds only., Less electronegative anions will result in a greater degree of covalency in the metal-anion bonds; this phenomenon was recognised and quantified by Shannon and co-authors in a series of papers by using a covalency parameter to indicate deviation from 'pure' ionic bonding. ${ }^{3,37,38}$ The influence of increasing covalency is that observed bond lengths are expected to be shorter than the sum of the two Shannon radii. The sum of the appropriate Shannon radii for a given bond shall henceforth be referred to here as the Shannon bond length, $D_{\text {Shannon}}$. For example, the $\mathrm{Pb}-\mathrm{F}$ Shannon bond length for octahedrally coordinated $\mathrm{Pb}$ is $D_{\text {Shannon }}(\mathrm{Pb}-\mathrm{F})=r_{\mathrm{Pb}(\mathrm{II})}+r_{\mathrm{F}^{-}}=1.19+1.285=$ $2.475 \AA$. If the variation in experimental interatomic distances compared to the Shannon bond length is due in whole or in part to increased covalency, then it is expected to be seen most prominently for the less electropositive metals of the $\mathrm{p}$ and $\mathrm{d}$ block, as for these compounds the difference in electronegativities between metal and anion, $\Delta \chi$, is small, so the degree of covalency is greater. The effect would be smaller for the $\mathrm{s}$ and $\mathrm{f}$ block metals.

To assess the applicability of Shannon radii to non-oxide/ fluoride compounds, and to quantify any deviation from $D_{\text {Shannon }}$ upon moving to heavier anions, a general survey was undertaken of experimental bond lengths in metal halide compounds, not limited to perovskite compounds. Given that the motivation for this work is predicting stable halide perovskite structures, we consider $\mathrm{M}-\mathrm{X}$ bond lengths where the metal $M$ is a candidate for the $\mathrm{B}$ site of the $\mathrm{ABX}_{3}$ halide perovskite structure. The perovskite B site is octahedrally coordinated by halide anions, so we limit our search to compounds containing $\mathrm{MX}_{6}$ octahedra, where $\mathrm{M}$ is a metal from a selection to be defined shortly, and $\mathrm{X}$ is a halide. The list of metals $\mathrm{M}$ to be considered was limited to those that could feasibly occupy the $\mathrm{B}$ site of such a perovskite, i.e. divalent metals for which $\mathrm{AMX}_{3}$ compounds are known and crystallographically characterised. This list of metals, M, used in this work is: $\mathrm{Mg}, \mathrm{Ca}, \mathrm{Ti}, \mathrm{V}, \mathrm{Cr}, \mathrm{Mn}$, Fe, Ni, Cd, Hg, Ge, Sn, Pb, Sm, Yb, Dy, Tm. Experimental room temperature and pressure structures were obtained from the 
ICSD using the CrystalWorks software. All compounds that include divalent metals from the list above, coordinated with exactly six of the same halide anions (and no other anions) were included. A total of 579 compounds were found matching the criteria set out above. Metal-halide distances for the first coordination sphere of the metal were calculated from the crystallographic information files. The experimental bond length, $D_{\text {obs }}(\mathrm{M}-\mathrm{X})$, was then taken as the mean of these metal-anion distances. Table S1 in the ESI $\uparrow$ shows the number of compounds used for calculation of the bond length for each metal-halide pair, and the standard deviation of bond lengths for each bond.

The deviation of $D_{\mathrm{obs}}(\mathrm{M}-\mathrm{X})$ from $D_{\text {Shannon }}$ was calculated and is plotted in Fig. 2. The Shannon bond length very closely approximates the measured bond lengths for all of the metal fluoride compounds considered. The $\mathrm{Hg}-\mathrm{F}$ bond showed the largest deviation of any metal considered here, the average $\mathrm{Hg}$ $\mathrm{F}$ bond distance was measured as $0.08 \AA$ shorter than the $\mathrm{Hg}-\mathrm{F}$ Shannon bond length, while all of the first row transition metal fluoride bonds were within $0.02 \AA$ of the Shannon bond length.

However, for $\mathrm{X}=\mathrm{Cl}, \mathrm{Br}$ and $\mathrm{I}$ the $\mathrm{M}-\mathrm{X}$ bonds for all metals considered (except $\mathrm{Mg}$ ) show considerable variation from the Shannon bond lengths. The d block metals typically show a shortening of their observed $\mathrm{M}-\mathrm{X}$ bond lengths compared with the Shannon bond length by $0.075-0.1 \AA$, with Ti, Cd and
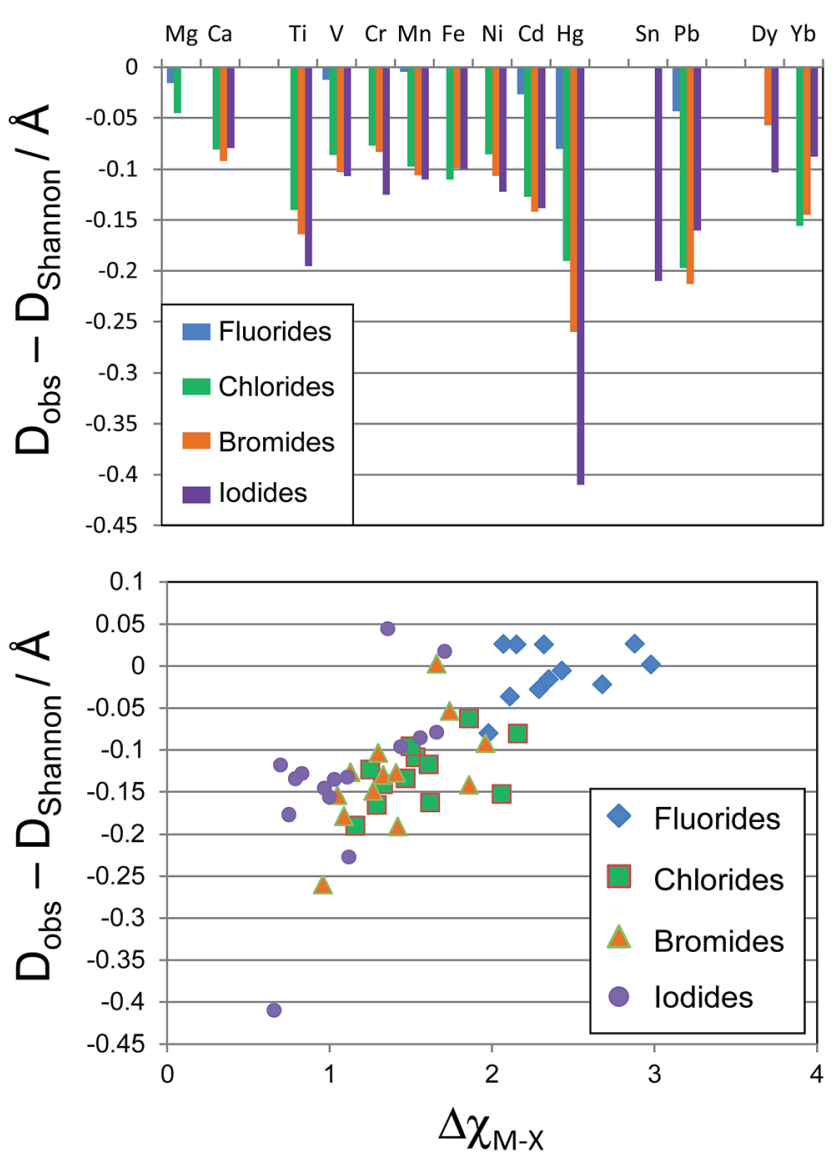

Fig. 2 Top, chart of deviation in experimental bond lengths from Shannon bond lengths in octahedral $M-X$ bonds ( $X=$ halide) by element. Bottom, plot of deviation in experimental bond lengths from Shannon bond lengths against difference in electronegativity.
$\mathrm{Hg}$ showing a considerably greater variation. For the d block metals the deviation tends to increase moving down the halide group from $\mathrm{Cl}$ to I. The p block post transition metals also show significant deviation from the Shannon bond lengths. Average $\mathrm{Pb}-\mathrm{Br}$ bonds are observed to be $0.21 \AA$ shorter than the $\mathrm{Pb}-\mathrm{Br}$ Shannon bond length. Sn-I bonds show a similar deviation from expected values.

If the deviation from Shannon bond length is due to increased covalency as has been suggested, then the effects should scale with the difference in electronegativity between the metal and halogen, $\Delta \chi_{\mathrm{M}-\mathrm{X}}$, as a smaller electronegativity difference tends to greater covalency. Fig. 2 shows a plot of $\Delta \chi_{\mathrm{M}-\mathrm{X}}$ against deviation from Shannon bond length for all $\mathrm{M}-\mathrm{X}$ bonds considered above. As can be seen, there is a correlation between the two variables, with smaller $\Delta \chi_{\mathrm{M}-\mathrm{X}}$ values tending to result in larger deviation from Shannon bond lengths. This suggests that the deviations are at least partially due to increased covalency in the $\mathrm{M}-\mathrm{X}$ bonds for the heavier halides. Some $\mathrm{M}-\mathrm{X}$ bonds do not seem to follow the general trend. For example the Mg-I and $\mathrm{Mg}-$ Br experimental bond length shows almost no variation from the Shannon bond length. This may be due to the chemical hardness of $\mathrm{Mg}^{2+}$, which is considerably greater than for other elements considered here: the Pearson hardness for $\mathrm{Mg}^{2+}, \eta=32.55 \mathrm{eV}$, while all of the d-block metals considered here have $\eta<10.3 \mathrm{eV}^{39}$ The $\mathrm{Hg}-\mathrm{X}$ bonds, however, show exceptionally large deviations: the $\mathrm{Hg}$-I bond length is over $0.4 \AA$ shorter than the Shannon bond length. This is despite the Pearson hardness of $\mathrm{Hg}^{2+}$ being close to those of the other $\mathrm{d}$ block metals considered here.

These deviations from the expected interatomic distances, howsoever caused, will influence the calculation of geometric ratios used to assess structure stability. Since the effect of contraction of bond lengths is both anion and cation dependent, it is not sufficient to apply an overall corrective factor to the existing Shannon radii, or to adjust the tolerance factor stability limits to account for the contraction. It is proposed here that for geometric calculations on heavier halide (Cl, Br, I) structures, a modified set of ionic radii be used. For our purposes of understanding the structural stability of halide perovskites, it is convenient to maintain the ionic radius of the halide anions at its standard Shannon value and introduce a new set of cation radii for metals in halide compounds, $r_{\mathrm{M}(\mathrm{x})}$, which depends, like the Shannon radii, on the metal, the charge state, the coordination environment, but unlike the Shannon radii, also on the halide to which the metal is bonded. These revised ratios are shown in Table 1 , together with the corresponding Shannon radii. To obtain $r_{\mathrm{M}(\mathrm{x})}$ values, the Shannon ionic radius of the appropriate halide anion $(1.285 \AA$ for fluoride, $1.85 \AA$ for chloride, $1.96 \AA$ for bromide, and $2.20 \AA$ for iodide) was subtracted from the average experimental bond length to yield the cation radius for each metal.

\section{Additional geometric considerations}

The tolerance factor alone, whether calculated using the revised radii in Table 1 or indeed the standard Shannon radii, is not sufficient for predicting the structures adopted by inorganic $\mathrm{ABX}_{3}$ compounds. In the following discussion, geometric ratios 
Table 1 Revised ionic radii used for halide compounds, compared with the corresponding Shannon radii. ${ }^{3} \mathrm{HS}=$ high spin

\begin{tabular}{|c|c|c|c|c|c|}
\hline \multirow[b]{2}{*}{ Cation } & \multirow[b]{2}{*}{$\begin{array}{l}\text { Six coordinate } \\
\text { Shannon ionic radius/Å }\end{array}$} & \multicolumn{4}{|c|}{ Experimental 6-coordinate cation radius, $r_{\mathrm{M}(\mathrm{X})}$} \\
\hline & & $\begin{array}{l}\text { Fluoride } \\
\text { compounds/A }\end{array}$ & $\begin{array}{l}\text { Chloride } \\
\text { compounds/A }\end{array}$ & $\begin{array}{l}\text { Bromide } \\
\text { compounds/Å }\end{array}$ & $\begin{array}{l}\text { Iodide } \\
\text { compounds/A }\end{array}$ \\
\hline $\operatorname{Mg}(\mathrm{II})$ & 0.72 & 0.70 & 0.67 & 0.72 & $0.75^{a}$ \\
\hline $\mathrm{Ca}(\mathrm{II})$ & 1.00 & 1.00 & $0.92^{a}$ & $0.91^{a}$ & 0.92 \\
\hline $\operatorname{Sr}(\mathrm{II})$ & 1.16 & - & - & - & 1.18 \\
\hline $\operatorname{Ti}(\mathrm{II})$ & 0.86 & - & 0.72 & 0.70 & 0.66 \\
\hline V(II) & 0.79 & 0.78 & 0.70 & 0.69 & 0.68 \\
\hline $\mathrm{Cr}(\mathrm{II})$ & $0.80(\mathrm{HS})$ & 0.82 & $0.72^{a}$ & $0.72^{a}$ & 0.68 \\
\hline $\operatorname{Mn}(\mathrm{II})$ & $0.83(\mathrm{HS})$ & 0.83 & 0.73 & 0.72 & 0.72 \\
\hline $\mathrm{Fe}(\mathrm{II})$ & $0.78(\mathrm{HS})$ & 0.80 & $0.67^{a}$ & 0.68 & $0.68^{a}$ \\
\hline $\mathrm{Ni}(\mathrm{II})$ & 0.69 & 0.71 & 0.60 & 0.58 & $0.57^{a}$ \\
\hline Cd(II) & 0.95 & $0.92^{a}$ & 0.82 & 0.81 & 0.81 \\
\hline $\mathrm{Hg}$ (II) & 1.02 & 0.94 & $0.83^{a}$ & 0.76 & $0.61^{a}$ \\
\hline Ge(II) & 0.73 & $b$ & $b$ & $b$ & 0.77 \\
\hline $\mathrm{Sn}$ (II) & $1.15^{c}$ & - & - & - & 0.97 \\
\hline $\mathrm{Pb}(\mathrm{II})$ & 1.19 & 1.15 & $0.99^{a}$ & 0.98 & 1.03 \\
\hline Tm(II) & 1.03 & - & $0.93^{a}$ & - & 0.95 \\
\hline $\operatorname{Sm}(\mathrm{II})$ & $1.22^{d}$ & $1.20^{a}$ & $1.02^{a}$ & 0.86 & $1.11^{a}$ \\
\hline $\mathrm{Yb}(\mathrm{II})$ & 1.02 & 1.05 & 0.86 & 0.88 & 0.93 \\
\hline Dy(II) & 1.07 & - & - & 1.01 & 0.97 \\
\hline
\end{tabular}

${ }^{a}$ Less than three crystallographically characterised compounds found, or standard deviation of experimental bond lengths above $0.1 \AA .{ }^{b}$ Ge(II) adopts highly distorted coordination environments so ionic radii not considered for $\mathrm{F}, \mathrm{Cl}, \mathrm{Br}$. ${ }^{c}$ Shannon does not give a Sn(II) radius, yet $1.15 \AA$ has been used by others. ${ }^{27} d$ No 6-coordinate Sm(II) radius is given by Shannon - the radius here is for 7 coordinate Sm(II). ${ }^{3,4}$ For statistical analysis see the ESI.

are calculated using the revised radii shown in Table 1 for the B site cations. The tolerance factor assesses whether the A cation can fit within the $\mathrm{BX}_{3}$ framework of corner sharing octahedra (referred to as the $\mathrm{ReO}_{3}$ structure) that is found in the cubic perovskite. However, another important consideration is whether the B site cation is of the correct size to be coordinated by six anions; i.e. whether the B site cation can fit in the octahedral hole in the anion sublattice. The radius of an octahedral hole, $r_{\text {hole }}$ formed within six close packed spheres of radius $r$ is:

$$
r_{\text {hole }}=0.41 r
$$

Therefore in the perovskite structure, assuming the hard sphere model for the ions, B site cations with radius smaller than $0.41 r_{\mathrm{X}}$ cannot be coordinated octahedrally without the anions overlapping. For the oxide and fluoride perovskites, the radius of the octahedral cavity ( $0.55 \AA$ and $0.52 \AA$ respectively) is such that only a few cations, for example $\mathrm{P}^{5+}, \mathrm{As}^{5+}$, and $\mathrm{Si}^{4+}$ are too small to fit within, and these cations are never found on the B site of oxide or fluoride perovskites. However, the octahedral cavity formed by six iodide anions is $0.90 \AA$ in radius, and many potential B site cations are smaller than this, as can be seen from Table 1. To assess the fit of the $\mathrm{B}$ site cation into the $\mathrm{X}_{6}$ octahedron, several authors have utilised the octahedral factor $\mu$ defined as: ${ }^{8}$

$$
\mu=\frac{r_{\mathrm{B}}}{r_{\mathrm{X}}}
$$

A plot of $t$ against $\mu$ can then be constructed and used as a structure map. Such a map assesses the suitability of both the A site cation and the B site cation for the perovskite structure.
Such a $t-\mu$ plot for the iodide perovskites is shown in Fig. 3. This will be discussed in detail since the iodide perovskites are the focus of current attention as potential new PV absorber materials. It can be seen that inorganic iodide perovskites,

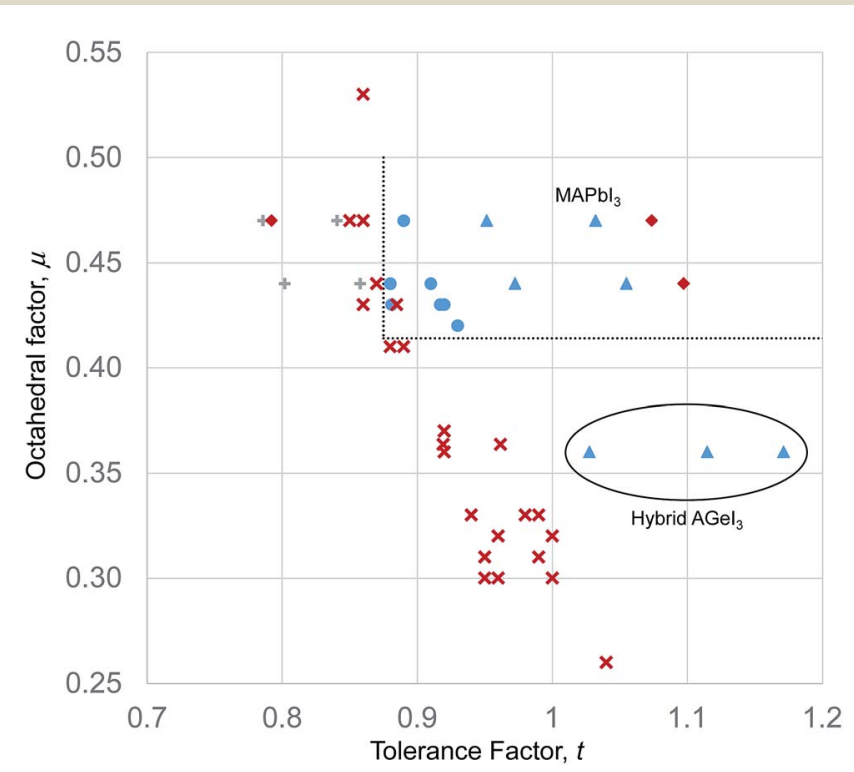

Fig. 3 Structural map of $\mathrm{ABI}_{3}$ compounds. Blue dots represent stable inorganic perovskites. Red crosses represent inorganic compounds that do not form perovskites. Blue triangles represent stable hybrid perovskites, whilst red diamonds represent hybrid compositions that do not form perovskites. Dotted lines are the boundary lines mentioned in the text. The anomalous hybrid Ge(॥) compounds are highlighted, as is $\mathrm{MAPbl}_{3}$. 
represented by blue circles, form in a distinct region of the structure map. This region is bounded by two well defined lines. A lower horizontal boundary line is at constant octahedral factor, with perovskites forming when $\mu>0.41$. Below this line, all known inorganic $\mathrm{ABI}_{3}$ compositions adopt non-perovskite structures. This limit corresponds closely to that seen for $\mathrm{ABO}_{3}$ compounds (where the limit was found to be $\mu>0.425$ ). ${ }^{7,40}$ Furthermore, it is encouraging that the boundary line found for the iodides corresponds exactly to the geometric limit for octahedral coordination of the B site of $\mu=0.41$, as this suggests that the revised cation radii derived in Table 1 are appropriate for describing these structures. A vertical boundary line is $t=0.875$. To the left (low tolerance factor) side of this line, six $\mathrm{ABI}_{3}$ compounds have been reported $\left(\mathrm{RbPbI}_{3}, \mathrm{RbSnI}_{3}\right.$, $\mathrm{KTmI}_{3}, \mathrm{TlPbI}_{3}, \mathrm{CsSrI}_{3}$ and $\mathrm{NH}_{4} \mathrm{PbI}_{3}$ ), and none of these form perovskites. As well as these non perovskites, several points representing examples of unknown or uncharacterised compositions made up of common elements $\left(\mathrm{NaPbI}_{3}, \mathrm{KSnI}_{3}\right.$, $\mathrm{NaSnI}_{3}$ ) are found to the left of this line, and are included on Fig. 3. The compound $\mathrm{KPbI}_{3}$, has been reported by several groups but no structure has been obtained, and there is uncertainty about the composition and even the colour. ${ }^{41-43}$ Therefore for the present this point is also marked as unknown.

The upper boundary in $\mu$, should there be one, is not well defined. There is only one reported $\mathrm{ABI}_{3}$ compound ( $\left.\mathrm{CsSrI}_{3}\right)$ which has $\mu>0.47$, and this is found at $\mu=0.53$, but in any case lies to the left of the vertical boundary line. Further synthetic efforts to produce compounds with higher octahedral factor are necessary to explore this region of the structure map and establish the true stability limits here.

The boundary of the stable perovskite region to the high tolerance factor side (right hand side as drawn in Fig. 1) is also not well defined, as there are no inorganic compounds with $t>$ 0.92 and $\mu>0.41$. To achieve a high tolerance factor, a large A site or a small B site cation is needed. The largest elemental cation in the periodic table (excluding radioactive elements) is $\mathrm{Cs}^{+}$, with radius $1.88 \AA$ in 12 coordination. Even if $\mathrm{Cs}^{+}$were combined with a hypothetical B site cation with exactly the radius necessary to meet the octahedral factor stability limit $\left(r_{\mathrm{B}}=0.41 r_{\text {iodide }}=0.41 \times 2.20 \AA=0.90 \AA\right)$, the resulting compound would have a tolerance factor of only 0.93. A smaller B site would lead to an octahedral factor too low for formation of perovskites. Thus for simple inorganic perovskites with $\mu>$ 0.41 , the maximum $t$ achievable is 0.93 , and to achieve a higher $t$ we must use complex cations as will be discussed in the following section.

For the inorganic iodide perovskites, the structural map and boundary lines set out above can separate the set of 32 known inorganic $\mathrm{ABI}_{3}$ structures successfully into perovskites and nonperovskites with only one compound miss-assigned. $\mathrm{RbYbI}_{3}$ sits inside the stable region of the structure map but is reported experimentally as a non-perovskite. $\mathrm{RBYbI}_{3}$ is close to the boundary of the stable region, and it may be that improved accuracy of the revised ionic radii will correct this.

Fig. 4 shows a combined structural map for all 159 halide perovskite considered here calculated with the revised cation radii from Table 1; the list of $\mathrm{ABX}_{3}$ compounds is that complied

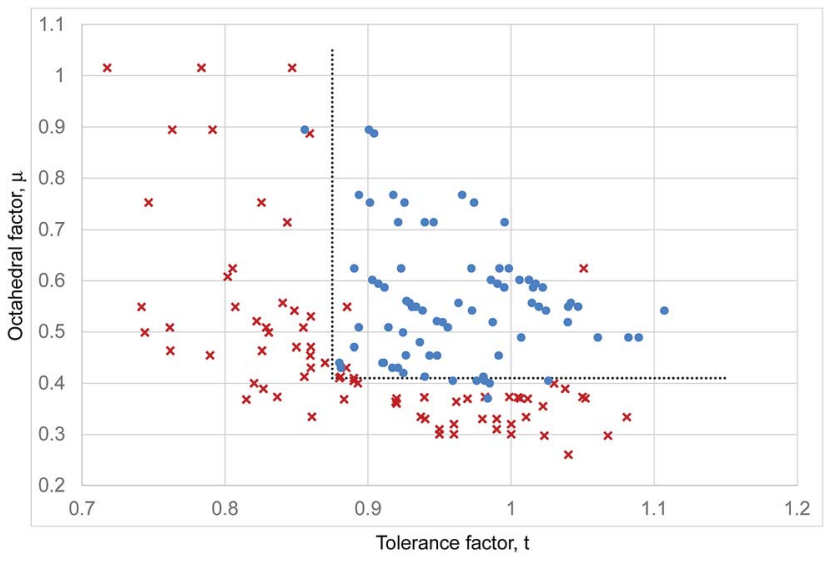

Fig. 4 Structural map of inorganic $\mathrm{ABX}_{3}$ compounds. Blue dots represent inorganic compositions that adopt the perovskite structure at room temperature and pressure. Red crosses represent inorganic compounds that do not form perovskites. The dotted lines represent the boundaries of the stable perovskite region as described in the text.

by Li et al. ${ }^{8}$ but treated with our revised ionic radii introduced above (Table S2, ESI $\dagger$ ). The same stability criteria as used for the iodides, $\mu>0.41$ and $t>0.875$, lead to $92 \%$ of the compositions considered being correctly determined as perovskites or nonperovskites. It can be seen that there is no defined upper boundary in octahedral factor, with examples of perovskite compounds with $\mu=0.89$, much higher than seen in the iodides.

Structural maps for the fluoride, chloride and bromide perovskites, constructed using the revised ionic radii from Table 1, are shown in the ESI (Fig. S1-S3†).

Based on octahedral factor criteria for perovskite stability, $\mu$ $>0.41$, there are a limited number of cations that may occupy a $\mathrm{B}$ site for a particular halide perovskite. This is an essential consideration when determining whether a particular $\mathrm{ABX}_{3}$ compound will form a perovskite structure. Those cations with $r_{\mathrm{M}(\mathrm{X})}<0.41 r_{\mathrm{X}}$ are too small to fit within the $\mathrm{X}_{6}$ octahedron. Table 2 shows a summary of the cations which give $\mu>0.41$ for each halide and therefore may be expected to be able to occupy the $\mathrm{B}$ site of fluoride, chloride, bromide and iodide perovskites. Since iodide has the largest $r_{\mathrm{X}}$ of the halides, only eight metals are sufficiently large to occupy the B site of an iodide perovskite: $\mathrm{Pb}$, $\mathrm{Sn}, \mathrm{Yb}, \mathrm{Dy}, \mathrm{Tm}, \mathrm{Sm}, \mathrm{Ca}, \mathrm{Sr}$. No inorganic A site cation is big enough to form $\mathrm{ASrI}_{3}$ or $\mathrm{ASmI}_{3}$ compounds with $t>0.875$, so these are not predicted to form inorganic perovskites. There are three inorganic iodide compounds that have $t$ and $\mu$ values within the stable perovskite region yet to our knowledge are so far unreported. These are $\mathrm{TlDyI}_{3}, \mathrm{TlYbI}_{3}$, and $\mathrm{TlTmI}_{3}$.

In summary, we demonstrate that an adapted geometric approach can categorise the room temperature structures of 31 out of 32 known inorganic iodide $\mathrm{ABI}_{3}$ compounds as perovskites or non-perovskites, and 147 correctly out of $159 \mathrm{ABX}_{3}(\mathrm{X}=$ $\mathrm{F}, \mathrm{Cl}, \mathrm{Br}, \mathrm{I})$ compositions. We introduce a revised set of cation radii for this task. These are anion specific and are calculated for divalent metals from the average six coordinate bond lengths of all compounds suitable compounds. The veracity of 
Table 2 List of metal cations that are predicted to be able to occupy the B site of halide perovskites, based on their revised anion dependent ionic radii listed in Table 1

\begin{tabular}{|c|c|c|c|c|}
\hline Anion & Fluoride & Chloride & Bromide & Iodide \\
\hline $\begin{array}{l}\text { Size of octahedral hole }= \\
0.41 r_{\mathrm{X}} / \AA\end{array}$ & 0.52 & 0.76 & 0.80 & 0.90 \\
\hline
\end{tabular}

these cation radii is demonstrated in several ways. Firstly they deviate from Shannon radii in a manner consistent with the degree of covalency expected due to the electronegativity (Fig. 2). Secondly, they allow construction of a structural map for the halide perovskites with stability limits based on geometric principles, specifically the octahedral factor limit which coincides with the geometric size of a hole within six close packed halide ions, with one single set of stability criteria for all halide perovskites.

\section{Application to hybrid iodide perovskites}

Hybrid perovskites as discussed here consist of an organic (usually a substituted ammonium) cation on the A site. In this discussion, we will include the ammonium ion $\left(\mathrm{NH}_{4}{ }^{+}\right)$itself along with organic A groups, although it is usually considered an inorganic ion. The hybrid iodide perovskites have recently become of great interest due to their exceptional photovoltaic properties. The following molecular cations have been successfully placed on the A site of an iodide perovskite: methylammonium $\left(\mathrm{CH}_{3} \mathrm{NH}_{3}\right.$, abbreviated here as $\left.\mathrm{MA}^{+}\right),{ }^{11}$ formamidinium $\left(\mathrm{H}_{2} \mathrm{~N}-\mathrm{CH}=\mathrm{NH}_{2}, \quad \mathrm{FA}^{+}\right)^{44}$ and acetamidinium $\left(\mathrm{CH}_{3} \mathrm{C}\left(\mathrm{NH}_{2}\right)_{2}{ }^{+}, \mathrm{AC}^{+}\right) .{ }^{45}$ From these, the following hybrid iodide perovskites with a single A site cation have been crystallographically characterised: $\mathrm{MAPbI}_{3}, \mathrm{MASnI}_{3}, \mathrm{MAGeI}_{3}, \mathrm{FAPbI}_{3}$, $\mathrm{FASnI}_{3}, \mathrm{FAGeI}_{3}$ and $\mathrm{ACGeI}_{3}$ (see Table S4 in ESI $\dagger$ for references for all iodide compounds). Mitzi has mentioned the successful synthesis of $\mathrm{MAEuI}_{3}$ (and $\mathrm{CsEuI}_{3}$ ), forming the perovskite structure ${ }^{46}$ but no crystallographic information could be found. Structures are known with mixed $\mathrm{FA}^{+}$and $\mathrm{MA}^{+}$cations on the $\mathrm{A}$ site. ${ }^{44}$ These would have $t$ and $\mu$ values intermediate between the pure A site compounds and are not considered further here.

The use of geometric ratios and stability maps is more challenging for hybrid perovskites than pure inorganic perovskites for a number of reasons which have already been discussed. In addition to the difficulties in representing the chemistry of these materials using geometric models, a further issue is that confidence in any system of structure prediction depends on a large number of data points. Only a small number of hybrid materials in the perovskite structure have been characterised, so it is difficult to assess the validity of any proposed system; a very large number of stability criteria will give perfect categorisation of the few existing materials, but that does not necessarily imply any physical basis for the criteria or any predictive power for unknown structures.
We now turn to the issue of quantifying the size of the A site cation in hybrid materials. Several approaches have been taken to model the size of molecular cations. Cheetham et al. quantified ammonium molecular ion radii, $r_{\text {Aeff }}$

$$
r_{\text {Aeff }}=r_{\text {mass }}+r_{\text {ion }}
$$

where $r_{\text {mass }}$ is the distance from the centre of mass of the molecule to the furthest non-hydrogen atom in the molecule, and $r_{\text {ion }}$ is the Shannon ionic radius of the nitride $\left(\mathrm{N}^{3-}\right)$ anion, which is $1.46 \AA .{ }^{26}$ The resulting ionic radii for ammonium $\left(\mathrm{NH}_{4}^{+}\right)$is $1.46 \AA$, methylammonium $\left(\mathrm{MA}^{+}\right)$is $2.16 \AA$, formamidinium $\left(\mathrm{FA}^{+}\right)$is $2.53 \AA$ and ethylammonium $\left(\mathrm{EA}^{+}\right)$is 2.74 $\AA$. Using these radii, and the revised $\mathrm{B}$ cation radii of Table 1 , the hybrid iodide perovskites are plotted on the structural map in Fig. 3. It can be seen that due to the larger size of the molecular A site cations, the position of the $\mathrm{MA}^{+}, \mathrm{FA}^{+}$and $\mathrm{EA}^{+}$ compounds lie to the right (high tolerance factor) side of the inorganic analogues - the octahedral factor is of course unchanged as it is not dependent on the A site cation. The $\mathrm{Pb}(\mathrm{II})$ and Sn(II) compounds fit well into the stability limits of the inorganic perovskites. $\mathrm{MAPbI}_{3}$ and $\mathrm{MASnI}_{3}$ have tolerance factors of 0.95 and 0.97 respectively. Both these compounds form perovskites, and in fact, $\mathrm{MASnI}_{3}$ is the only known iodide compound to form in the undistorted cubic perovskite structure at room temperature and pressure. This correlates well with its tolerance factor being the closest to 1 of any iodide compound, inorganic or hybrid, considered here. $\mathrm{FAPbI}_{3}$ and $\mathrm{FASnI}_{3}$ have tolerance factors of 1.03 and 1.06 using the revised ionic radii. Both these compounds form perovskites, indicating that, as with the fluoride perovskites, ${ }^{8}$ tolerance factors above 1 can result in perovskite compounds. $\mathrm{EAPbI}_{3}$ and $\mathrm{EASnI}_{3}$ have tolerance factors of 1.07 and 1.10 respectively. Neither of these compounds form perovskites, and therefore it appears that the high tolerance factor limit for hybrid iodide perovskites is between 1.06 and 1.07. One compound with $\mathrm{A}=\mathrm{NH}_{4}{ }^{+}$has been characterised: $\mathrm{NH}_{4} \mathrm{PbI}_{3}$, has a tolerance factor of 0.79 and lies outside the stable region for perovskites in Fig. 3, and indeed this compound does not form a perovskite structure at ambient conditions. ${ }^{47}$

Additionally, several hybrid perovskite compounds with Ge(II) on the B site have been reported, and these do not fit the stability limits shown in Fig. 3 and described above. $\mathrm{MAGeI}_{3}$, $\mathrm{FAGeI}_{3}$ and $\mathrm{ACGeI}_{3}$ were synthesised by Kanatzidis et al. ${ }^{45}$ The latter compound includes the acetamidinium $\mathrm{CH}_{3} \mathrm{C}\left(\mathrm{NH}_{2}\right)_{2}{ }^{+}$ cation, the radius of which has not been previously calculated 
using the procedure of Cheetham et al. The crystal structure of acetamidinium chloride indicates each $\mathrm{C}-\mathrm{N}$ bond is $1.305 \AA{ }^{48}$ and making the simplification of considering the centre of mass to be located at the central carbon atom, we can take $r_{\text {mass }}=$ $1.305 \AA$. According to eqn (4), $r_{\text {Aeff }}$ for the $\mathrm{AC}^{+}$ion is therefore $2.77 \AA$, significantly larger than $\mathrm{MA}^{+}$or $\mathrm{FA}^{+}$. That $\mathrm{MAGeI}_{3}$, $\mathrm{FAGeI}_{3}$ and $\mathrm{ACGeI}_{3}$ form perovskites is surprising, given the octahedral factor significantly smaller than the geometric limit of 0.41 , and, in addition, the perovskite $\mathrm{ACGeI}_{3}$ has a very large tolerance factor of 1.17. All of the aforementioned Ge(II) compounds reported by Kanatzidis et al. display highly distorted $\mathrm{GeI}_{6}$ octahedra, with three short and three much longer Ge-I bonds. ${ }^{45}$ This is attributed by the authors to a stereoactive lone pair on the Ge(II) centre, and may explain how, in this series of compounds, the octahedral factor and tolerance factor requirements can be relaxed. Only group 14 metals may have stereochemically active lone pairs in the +2 state, meaning this phenomenon will not be widespread and might be seen as an exception to the established stability rules. It may also be that these Ge(II) compounds, which are formed using low temperature routes, are kinetic products and more thermodynamically stable configurations are possible.

From the results above, we tentatively assign a limit to the stable perovskite region at $t \leq 1.06$. Whilst the stability limits for the iodide perovskites could be defined with some confidence due to the relatively large number of compounds available, any such limits applied to the hybrid perovskites must be less certain due to the smaller number of compounds available to test the model, as well as the challenge the hybrid structure presents to the assumption of the hard sphere model.

The unusual stability of the Ge(II) compounds may be related solely to the ability of Ge(II) to adopt highly distorted coordination due to a stereoactive lone pair. Thus, leaving aside possible Ge(II) compounds, which do not seem to be predictable using the methods employed here, we show in Table 3 our predictions of unreported compounds that will be stable perovskites, all of which have $\mu>0.41$ and $t \leq 1.06$. We consider

Table 3 List of unreported hybrid iodide $\mathrm{ABI}_{3}$ compounds that fall within the stable region of the structural map

\begin{tabular}{lll}
\hline Compound & $\begin{array}{l}\text { Tolerance factor, } \\
t\end{array}$ & $\begin{array}{l}\text { Octahedral } \\
\text { factor, } \mu\end{array}$ \\
\hline $\mathrm{MADyI}_{3}$ & 0.97 & 0.44 \\
$\mathrm{FADyI}_{3}$ & 1.06 & 0.44 \\
$\mathrm{MASmI}_{3}$ & 0.93 & 0.50 \\
$\mathrm{FASm}_{3}$ & 1.01 & 0.50 \\
$\mathrm{EASm}_{3}$ & 1.05 & 0.50 \\
$\mathrm{ACSm}_{3}$ & 1.06 & 0.50 \\
$\mathrm{MATmI}_{3}$ & 0.98 & 0.43 \\
$\mathrm{FATmI}_{3}$ & 1.06 & 0.43 \\
$\mathrm{MAYbI}_{3}$ & 0.98 & 0.43 \\
$\mathrm{MACaI}_{3}$ & 0.99 & 0.42 \\
$\mathrm{MASrI}_{3}$ & 0.92 & 0.53 \\
$\mathrm{FASr}_{3}$ & 1.00 & 0.53 \\
$\mathrm{EASrI}_{3}$ & 1.04 & 0.53 \\
$\mathrm{ACSrI}_{3}$ & 1.05 & 0.53
\end{tabular}

only the $\mathrm{NH}_{4}^{+}, \mathrm{MA}^{+}, \mathrm{FA}^{+}, \mathrm{AC}^{+}$and $\mathrm{EA}^{+}$cations, as these have been experimentally incorporated into $\mathrm{ABI}_{3}$ structures.

Interestingly, several EA and AC compounds are predicated to be stable as perovskites. However, while these compounds may be stable, the $\mathrm{B}$ site cations used $\left(\mathrm{Sr}^{2+}\right.$ and $\left.\mathrm{Sm}^{2+}\right)$ are likely to have very different contributions to the electronic structure compared with $\mathrm{Pb}^{2+}$ and $\mathrm{Sn}^{2+}$ that have so far formed the most successful hybrid PV materials. The stability of $\mathrm{CH}_{3} \mathrm{NH}_{3} \mathrm{SrI}_{3}$ in the perovskite structure has recently been predicted by DFT, in agreement with our analysis here. ${ }^{28}$ However, the band gap is calculated as $3.6 \mathrm{eV}$, far too high for use as a PV absorber. We have attempted synthesis of $\mathrm{MADyI}_{3}, \mathrm{MAYbI}_{3}, \mathrm{MATmI}_{3}$ and found the products to be highly moisture sensitive, which has so far precluded a definite structural determination. Whilst isolation of phase pure samples is no doubt possible, it is likely that their instability may prevent technological application in solar cells.

The approach adopted here relates to $\mathrm{ABX}_{3}$ hybrid compounds. The related series of alkylammonium metal formates, $\mathrm{AB}(\mathrm{HCOO})_{3}$, can be successfully treated by a tolerance factor approach using the traditional Shannon radii. ${ }^{27}$

\section{Conclusions}

We propose an adaptation to the traditional tolerance factor approach for use with halide perovskites in general and hybrid iodide perovskites in particular. Using revised ionic radii, that take into account greater covalency in some metal-halide bonds, and a structure map approach, a system of classification can be devised that can correctly categorise 31 out of 32 inorganic iodide perovskites, and 147 out of $159 \mathrm{ABX}_{3}(\mathrm{X}=\mathrm{F}, \mathrm{Cl}, \mathrm{Br}$, I) compositions. Such a system also seems to apply to the hybrid perovskites, although the Ge(II) compounds are clear exceptions due to their stereoactive lone pair. We conclude that only a handful of cations may be successfully placed on the B site of an iodide perovskite: $\mathrm{Pb}, \mathrm{Sn}, \mathrm{Yb}, \mathrm{Dy}, \mathrm{Tm}, \mathrm{Sm}, \mathrm{Ca}, \mathrm{Sr}$. The $\mathrm{Pb}$ and Sn containing hybrid iodide perovskite compounds are very well studied, and we report here that Dy, Tm and $\mathrm{Yb}$ hybrid iodide perovskites appear highly moisture sensitive. The $\mathrm{Ca}$ and $\mathrm{Sr}$ compounds are unlikely to show the narrow band gaps required for PV applications due to the electronic differences between group 2 metals and post transition metals. ${ }^{28}$ There is still more work to do to explore the list of compounds in Table 3, but overall we feel that while there is still great scope for optimisation of existing materials, there may be little opportunity for discovery of entirely new, effective hybrid solar absorber perovskite materials. The search for further hybrid solar absorber materials may therefore have to extend beyond the simple perovskite structure, whether that is towards recently reported double perovskites, ${ }^{49}$ or to more diverse hybrid structures.

\section{Acknowledgements}

RGP, DOS and HB acknowledge flexible funding from and membership of the EPSRC SuperSolar Hub. RGP and DOS are members of the Materials Design Network. 


\section{Notes and references}

1 A. Walsh, Nat. Chem., 2015, 7, 274-275.

2 S. M. Woodley and R. Catlow, Nat. Mater., 2008, 7, 937-946.

3 R. D. Shannon, Acta Crystallogr., Sect. A: Cryst. Phys., Diffr., Theor. Gen. Crystallogr., 1976, 32, 751-767.

4 R. D. Shannon and C. T. Prewitt, Acta Crystallogr., Sect. B: Struct. Crystallogr. Cryst. Chem., 1969, 25, 925-946.

5 V. M. Goldschmidt, Naturwissenschaften, 1926, 14, 477-485.

6 A. S. Bhalla, R. Y. Guo and R. Roy, Mater. Res. Innovations, 2000, 4, 3-26.

7 C. Li, K. C. K. Soh and P. Wu, J. Alloys Compd., 2004, 372, 4048.

8 C. Li, X. Lu, W. Ding, L. Feng, Y. Gao and Z. Guo, Acta Crystallogr., Sect. B: Struct. Sci., 2008, 64, 702-707.

9 A. Kojima, K. Teshima, Y. Shirai and T. Miyasaka, J. Am. Chem. Soc., 2009, 131, 6050-6051.

10 B. E. Hardin, H. J. Snaith and M. D. McGehee, Nat. Photonics, 2012, 6, 162-169.

11 F. Hao, C. C. Stoumpos, D. H. Cao, R. P. H. Chang and M. G. Kanatzidis, Nat. Photonics, 2014, 8, 489-494.

12 P. P. Boix, S. Agarwala, T. M. Koh, N. Mathews and S. G. Mhaisalkar, J. Phys. Chem. Lett., 2015, 6, 898-907.

13 M. Safdari, A. Fischer, B. Xu, L. Kloo and J. M. Gardner, J. Mater. Chem. A, 2015, 3, 9201-9207.

14 A. Walsh, J. Phys. Chem. C, 2015, 119, 5755-5760.

15 M. M. Lee, J. Teuscher, T. Miyasaka, T. N. Murakami and H. J. Snaith, Science, 2012, 338, 643-647.

16 J.-H. Im, J. Chung, S.-J. Kim and N.-G. Park, Nanoscale Res. Lett., 2012, 7, 353.

17 K. Chondroudis and D. B. Mitzi, Chem. Mater., 1999, 11, 3028-3030.

18 J. L. Knutson, J. D. Martin and D. B. Mitzi, Inorg. Chem., 2005, 44, 4699-4705.

19 I. Borriello, G. Cantele and D. Ninno, Phys. Rev. B: Condens. Matter Mater. Phys., 2008, 77, 235214.

20 A. K. Cheetham, C. N. R. Rao and R. K. Feller, Chem. Commun., 2006, 4780-4795, DOI: 10.1039/B610264F.

21 E. Beurer, J. Grimm, P. Gerner and H. U. Guedel, Inorg. Chem., 2006, 45, 9901-9906.

22 I. Chung, J.-H. Song, J. Im, J. Androulakis, C. D. Malliakas, H. Li, A. J. Freeman, J. T. Kenney and M. G. Kanatzidis, J. Am. Chem. Soc., 2012, 134, 8579-8587.

23 M. Zhuravleva, B. Blalock, K. Yang, M. Koschan and C. L. Melcher, J. Cryst. Growth, 2012, 352, 115-119.

24 A. C. Lindsey, M. Zhuravleva, L. Stand, Y. Wu and C. L. Melcher, Opt. Mater., 2015, 48, 1-6.

25 J. Berry, T. Buonassisi, D. A. Egger, G. Hodes, L. Kronik, Y.-L. Loo, I. Lubomirsky, S. R. Marder, Y. Mastai, J. S. Miller, D. B. Mitzi, Y. Paz, A. M. Rappe, I. Riess,
B. Rybtchinski, O. Stafsudd, V. Stevanovic, M. F. Toney, D. Zitoun, A. Kahn, D. Ginley and D. Cahen, Adv. Mater., 2015, 27, 5102-5112.

26 G. Kieslich, S. Sun and A. K. Cheetham, Chem. Sci., 2014, 5, 4712-4715.

27 G. Kieslich, S. Sun and A. K. Cheetham, Chem. Sci., 2015, 6, 3430-3433.

28 T. J. Jacobsson, M. Pazoki, A. Hagfeldt and T. Edvinsson, J. Phys. Chem. C, 2015, 119, 25673-25683.

29 C. K. Moller, Nature, 1958, 182, 1436.

30 C. C. Stoumpos, C. D. Malliakas and M. G. Kanatzidis, Inorg. Chem., 2013, 52, 9019-9038.

31 D. M. Trots and S. V. Myagkota, J. Phys. Chem. Solids, 2008, 69, 2520-2526.

32 C. Hohnstedt and G. Meyer, Z. Anorg. Allg. Chem., 1993, 619, 1374-1378.

33 G. Schilling, C. Kunert, T. Schleid and G. Meyer, Z. Anorg. Allg. Chem., 1992, 618, 7-12.

34 M. Suta and C. Wickleder, J. Mater. Chem. C, 2015, 3, 52335245.

35 G. Thiele and B. R. Serr, Zeitschrift für Kristallographie, 1995, 210, 64.

36 S. H. Wang and M. Zhoa, J. Less Common Met., 1987, 127, 219-224.

37 R. D. Shannon, P. S. Gumerman and J. Chenavas, Am. Mineral., 1975, 60, 714-716.

38 R. D. Shannon and P. S. Gumerman, J. Inorg. Nucl. Chem., 1976, 38, 699-703.

39 R. G. Pearson, Inorg. Chem., 1988, 27, 734-740.

40 L. M. Feng, L. Q. Jiang, M. Zhu, H. B. Liu, X. Zhou and C. H. Li, J. Phys. Chem. Solids, 2008, 69, 967-974.

41 A. Cortel, J. Chem. Educ., 1997, 74, 297.

42 T. A. Kuku and A. M. Salau, Solid State Ionics, 1987, 25, 1-7. 43 O. N. Yunakova, V. K. Miloslavsky and E. N. Kovalenko, Opt. Spectrosc., 2014, 116, 68-71.

44 G. E. Eperon, S. D. Stranks, C. Menelaou, M. B. Johnston, L. M. Herz and H. J. Snaith, Energy Environ. Sci., 2014, 7, 982-988.

45 C. C. Stoumpos, L. Frazer, D. J. Clark, Y. S. Kim, S. H. Rhim, A. J. Freeman, J. B. Ketterson, J. I. Jang and M. G. Kanatzidis, J. Am. Chem. Soc., 2015, 137, 6804-6819.

46 D. B. Mitzi, in Progress in Inorganic Chemistry, ed. K. D. Karlin, John Wiley \& Sons Inc, New York, 1999, vol. 48, pp. 1-121.

47 L.-Q. Fan and J.-H. Wu, Acta Crystallogr., Sect. E: Struct. Rep. Online, 2007, 63, i189-U144.

48 J. R. Cannon, A. H. White and A. C. Willis, J. Chem. Soc., Perkin Trans. 2, 1976, 271-272, DOI: 10.1039/p29760000271.

49 E. T. McClure, M. R. Ball, W. Windl and P. M. Woodward, Chem. Mater., 2016, 28, 1348-1354. 